\title{
A complex systems methodology to transition management
}

\author{
Floortje Alkemade • Koen Frenken • \\ Marko P. Hekkert • Malte Schwoon
}

Published online: 13 May 2009

(C) Springer-Verlag 2009

\begin{abstract}
There is a general sense of urgency that major technological transitions are required for sustainable development. Such transitions are best perceived as involving multiple transition steps along a transition path. Due to the path dependent and irreversible nature of innovation in complex technologies, an initial transition step along some preferred path may cut off paths that later may turn out to be more desirable. For these reasons, initial transition steps should allow for future flexibility, where we define flexibility as robustness regarding changing evidence and changing preferences. We propose a technology assessment methodology based on rugged fitness landscapes, which identifies the flexibility of initial transition steps in complex technologies. We illustrate our methodology by an empirical application to 2,646 possible future car systems.
\end{abstract}

Keywords NK-model • Complexity • Flexibility • Irreversibility •

Path dependence - Transition path - Transition management •

Sustainable development • Car technology

F. Alkemade - M. P. Hekkert

Faculty of Geosciences, Copernicus Institute for Sustainable Development \& Innovation,

Utrecht University, Utrecht, The Netherlands

\section{K. Frenken $(\bowtie)$}

Faculty of Geosciences, Urban \& Regional Research Centre Utrecht (URU),

Utrecht University, Utrecht, The Netherlands

e-mail: k.frenken@geo.uu.nl

M. Schwoon

Statkraft Markets GmbH, Düsseldorf, Germany

M. Schwoon

International Max Planck Research School on Earth System Modelling, Hamburg, Germany 
JEL Classification $\mathrm{C} 15 \cdot \mathrm{D} 83 \cdot \mathrm{O} 32 \cdot \mathrm{Q} 01 \cdot \mathrm{Q} 42 \cdot \mathrm{Q} 55$

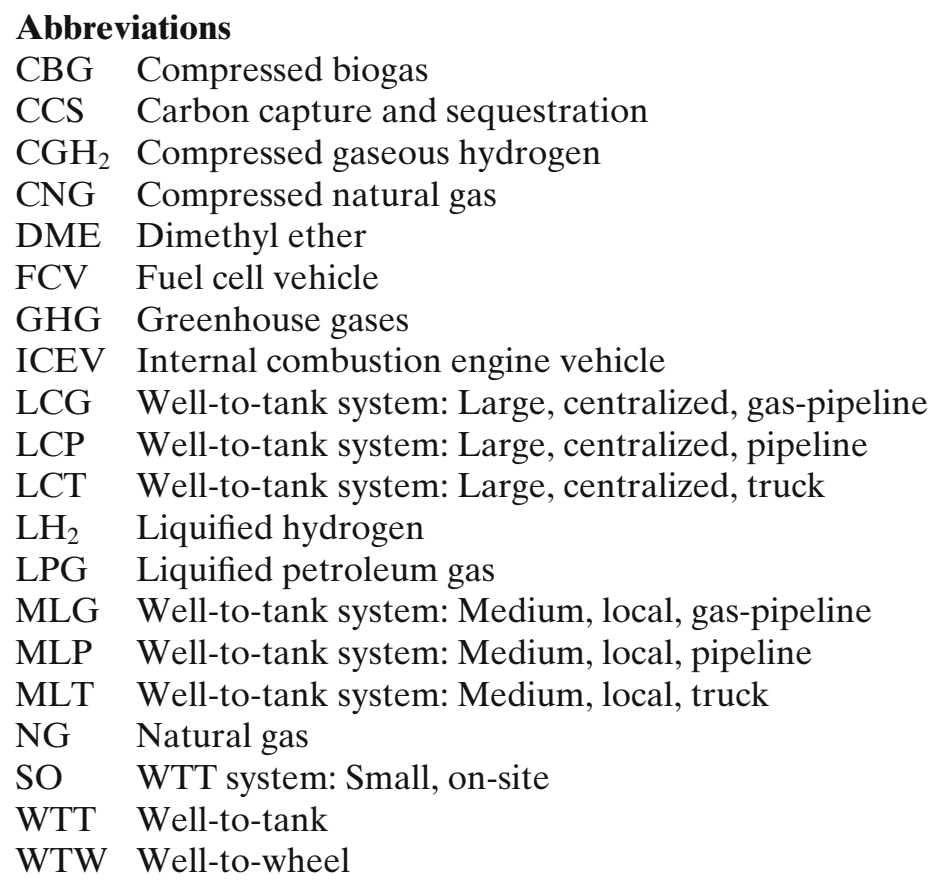

\section{Introduction}

Technology assessment traditionally focuses on comparing the desirability of alternative technological options. In such a perspective, the transition path to be followed in the future is dictated by the alternative that is preferred at present. This approach to technological transitions faces two problems. First, the future performance of alternative technological systems is uncertain. Due to the path dependent and irreversible nature of technological development (David 1985; Arthur 1989; Cowan 1990), initial transition steps along a particular path may cut off alternative paths, which may turn out to be more desirable at a future moment in time. This means that, if new information becomes available that favors an alternative technological system, it will be difficult to re-orient the transition path into a new direction. Second, societal preferences may change during a transition process (Pinch and Bijker 1984). Such a change may induce a reversal of the transition process wasting time and resources. For these reasons, we argue that initial transition steps should be flexible, where we define a flexible transition strategy as a strategy that is robust with regard to changing evidence and changing preferences.

Transitions in large technical systems such as transport and energy technologies are characterized by irreversibility and path dependence. Changes in such systems often involve high sunk costs causing such investments to be 
expensive to reverse. To take into account this path dependence and irreversibility inherent to technological transitions, we propose a complex systems approach to technology assessment based on the concept of rugged fitness landscapes (Kauffman 1993). Complex technological systems are characterized by rugged fitness landscapes with local optima reflecting compromises between conflicting constraints. In this framework, flexibility can be defined in two ways. First, initial transition steps should be robust in the case of changing evidence regarding the 'fitness' (performance) of alternative technological options. Changing evidence can be dealt with by maximizing the number of local optima that can still be reached after an initial transition step and by maximizing the number of possible paths towards each local optimum after an initial transition step has been taken. Second, initial transition steps should be robust to changing preferences to avoid a reversal of the transition process. Changing preferences can be dealt with by pursuing an initial transition step that yields an improvement regarding all preferences (Pareto improvement).

We will proceed as follows. Section 2 describes the complex systems model based on rugged fitness landscapes. We will explain the logic underlying rugged fitness landscapes using a simple simulation using the NK algorithm (Kauffman 1993). Section 3 introduces the two notions of flexibility regarding initial transition steps using the example of NK fitness landscapes. In Section 4, we apply the flexibility measures to empirical data describing all possible transition paths in a design space comprising 2,646 alternative car systems. We emphasize that the application to car technology data serves an illustrative purpose more than a predictive purpose. Section 5 concludes.

\section{NK fitness landscapes}

Complex technological systems contain several interdependent subsystems that function in a coherent manner (Rosenberg 1969; Simon 1969; Hughes 1983; Vincenti 1990; Silverberg and Verspagen 2005). Interdependencies between subsystems render the performance or fitness of the overall system dependent on the specific combination of the subsystems. All possible combinations form the state space or design space of the technological system. The combinatorial logic of assembling systems from subsystems implies that the number of possible designs that can be assembled from only a small set of subsystems is large. For example, a system with only ten elements, each of which can be designed in two ways, has a design space of $2^{10}=1,024$ possible designs. Depicting technological change as a search process within a design space captures the idea that future technological systems can be represented as combinations of known subsystems. Empirical studies of technological change have shown that many innovations indeed occurred through the combination of existing subsystem technologies (Frenken 2006). Complex systems theory provides us with models to study the effects of interdependencies among subsystems on combinatorial search processes. 
Fig. 1 A design space with $N=3, K=2$ and fitness function $f$

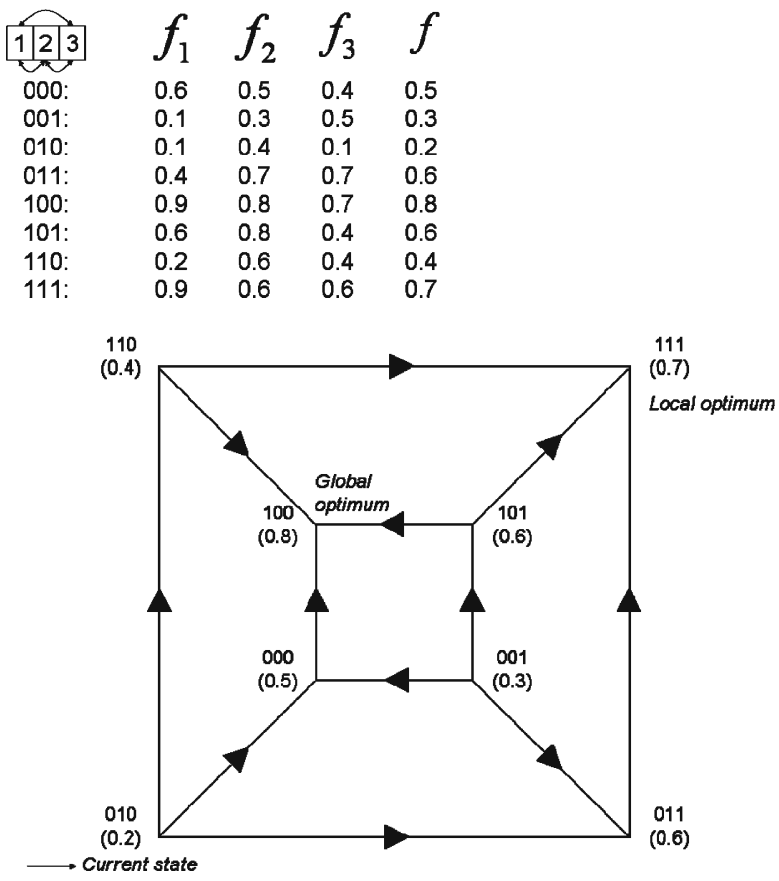

One influential model is the $N K$-model (Kauffman 1993). ${ }^{1}$ We introduce this model here for didactic purposes, yet it is important to recognize that our empirical methodology does not depend on the assumptions underlying the NK-model. The complexity of a system with $N$ subsystems is indicated by $K$ that refers to the number of dependency relations between elements. The possible value of $K$ ranges from its minimum $K=0$ to its maximum $K=$ $N-1$. If the performance of subsystems is not affected by other subsystems, we have $K=0$, while if the performance of subsystems is affected by all other subsystems, we have $K=N-1$. In the case of minimum complexity, the fitness of each subsystem is determined independently from the state of other subsystems, while in the case of maximum complexity, the fitness of each subsystem is different for each different combination of subsystems. Note that real-world systems will typically be characterized by intermediate levels of complexity (Simon 2002).

Figure 1 gives an example of a design space of a technological system with three subsystems $(N=3)$, where each subsystem can be designed in two different ways. The total number of states in this design space amounts to $2^{3}=8$ technology designs. The fitness of each particular subsystem is dependent on the choice of the other two subsystems $(K=2)$, as the fitness of each subsystem is different depending on the choice of design for the other

\footnotetext{
${ }^{1}$ For a review of NK-models in the context of technological innovation, see Frenken (2006).
} 
subsystems. ${ }^{2}$ Following Kauffman (1993), the fitness value for each subsystem in each specific combination of subsystems can be simulated by random draws from a uniform distribution between 0 and 1 . We thus need to draw three random numbers for each of the eight different designs as is done in Fig. 1. The fitness of the system as a whole can then be derived as the mean of the fitness values of the subsystems. ${ }^{3}$ In Fig. 1 , state 100 is a global optimum and state 111 is a local optimum. Global and local optima in the design space correspond to peaks in the fitness landscape (hence the metaphor of rugged fitness landscapes). A global optimum is a local optimum that has a superior fitness compared to all other designs, while a local optimum is a design that is superior compared to its neighboring designs, but not compared to all possible designs. Thus, a global optimum is also a local optimum, while a local optimum is not necessarily globally optimal.

Having specified a design space and the corresponding fitness values (the 'fitness landscape'), one can understand technological change as a move from one design $x$ to a new design $y$ in the design space. We make two assumptions. First, we assume that only one subsystem is allowed to change at each time, which means that each subsystem innovation can be represented as a move to a 'neighboring' design along one of the axes in the design space. The assumption that technological transitions consist of a series of changes in subsystems is supported by the observation that, at least for large technological systems, a change in a single subsystem is very costly and can easily take 5-10 years to complete. Second, we assume that a move in design space only occurs if this move does not decrease the fitness $f$ of a technological system, hence, the metaphor of 'hill-climbing' (Kauffman 1993). Since a change in one subsystem is very costly and time-consuming, one can expect any subsystem change that decreases fitness to be socially unacceptable (thus excluding the strategy of 'one step back, two steps forward'). This assumption is supported by the observation that changes in any subsystem often involve durable investments in infrastructure which make these changes expensive to reverse. In the following, we will call each innovation in a single subsystem a transition step, and a series of transition steps a transition path.

The shape of the fitness landscape determines the possible transition paths. In the simulation example, once a series of innovations ends up in local optimum 111, it becomes impossible to make the transition to the global optimum 100. A transition towards a local optimum is irreversible, as any move away from a local optimum would imply a decrease in performance. A transition path may thus well become 'locked in' into a local optimum. Transition paths are also characterized by path dependence. For example, if

\footnotetext{
${ }^{2}$ An implicit assumption of the NK-model holds that the number of subsystems equals the number of fitness values. This assumption can be removed in a generalized NK-model without changing the logic of fitness landscapes (Altenberg 1994).

${ }^{3}$ In the context of technological innovation, system elements correspond to the technical characteristics of a design and the fitness values to service characteristics (Saviotti and Metcalfe 1984).
} 
the first transition step is $010 \rightarrow 011$, the only remaining transition step from 011 is $011 \rightarrow 111$. An alternative transition path starts from the transition step $010 \rightarrow 000$, from which the only second step is $000 \rightarrow 100$. A myopic search process is thus path dependent in the sense that early decisions determine the scope of future decisions. Figure 1 also shows that there can be multiple paths to the same optimum. In the example, the local optimum can be reached in two ways: via $010 \rightarrow 011 \rightarrow 111$ and via $010 \rightarrow 110 \rightarrow 111$. The global optimum can also be reached in two ways: via $010 \rightarrow 000 \rightarrow 100$ and via $010 \rightarrow 110 \rightarrow 100$. Fitness landscapes for larger design spaces will typically be characterized by many possible uphill paths towards optima of different lengths, a property that we will explore below.

\section{Flexibility in technological transitions}

We will now use the properties of rugged fitness landscapes to derive three flexibility measures that can be used to assess the desirability of the first transition step while taking into account future uncertainties. The first two measures are based on uncertainty regarding current evidence. An important source of uncertainty in technology assessment is whether the fitness assigned to each possible design in design space is accurate. Forecasting models are necessarily based on current assumptions about technological progress, which later may turn out to have been misinformed. History is full of examples in which commonly held expectations regarding future technological progress turned out to be plain wrong. ${ }^{4}$

The flexibility of a first transition step can be expressed in terms of the number of options it leaves open if evidence about fitness changes over time. In the following, we define changing evidence as evidence that a particular design $s$, which previously was thought to have some fitness $f$, actually has zero fitness. This is a stylized way to say that the changing evidence convinces the decision-maker no longer to pursue any transition path that involves design $s$.

If changing evidence concerns an optimum towards which the search process is heading, flexibility is desired so as to be able to reorient the search process towards an alternative optimum. Consider again Fig. 1. If a transition path is chosen towards the global optimum 100 , transition path $010 \rightarrow 000 \rightarrow 100$ would normally be preferred over $010 \rightarrow 110 \rightarrow 100$, because the fitness of the intermediate design is higher for $010 \rightarrow 000 \rightarrow 100(f(000)=0.5)$ than for $010 \rightarrow 110 \rightarrow 100(f(110)=0.4)$. However, after $010 \rightarrow 000$, the only remaining transition step is $000 \rightarrow 100$, while after $010 \rightarrow 110$, one can still choose between $110 \rightarrow 100$ or $110 \rightarrow 111$. This means that, if new evidence becomes available after the first transition step $010 \rightarrow 000$, that design 100 has zero fitness, the

\footnotetext{
${ }^{4}$ To give one example of a completely wrong prediction is the commonly held prediction that in the post-war US, every household would soon have a family helicopter (Taylor 1995: 164).
} 
transition process gets stuck at 000 . The alternative transition path to 100 via 110, however, can still be reoriented towards local optimum 111 if the fitness of 000 turns out to be zero. We can thus define flexibility according to this principle of 'no regret' as the number of different optima that can be reached after the initial transition step has taken place. We will call this type of flexibility design flexibility. In the example in Fig. 1, transition steps $010 \rightarrow 000$ and $010 \rightarrow 011$ have design flexibility equal to 1 and the transition step $010 \rightarrow 110$ has design flexibility equal to 2 .

Another way to explain design flexibility is in graph-theoretical terms. A fitness landscape can be represented as a network (graph) with each node representing a design and each directed link (arc) representing an up-hill move from one design to a neighboring design. The design flexibility of a node equals the number of local optima that can be reached following a path of directed links. The Appendix provides the formal expression.

If the changing evidence does not regard the fitness of an optimum, but of designs that lie on a transition path towards an optimum, the transition path towards an optimum becomes blocked at some point along the path. This is implied by the assumption that an innovation is only accepted if it does not decrease fitness. If, after the initial transition step, new evidence becomes available that the next transition step would actually lead to zero fitness, the transition process comes to an end, unless there are alternative paths towards an optimum. The number of alternative paths towards an optimum can thus be considered a flexibility measure. The second flexibility measure, which we call path flexibility, therefore counts the number of different paths that lead to an optimum given an initial transition step. In the simulation in Fig. 1, the path flexibility of all three initial transition steps regarding any optima that can be reached after the initial step, equals 1. A second simulation provided in Fig. 2 shows an example of a fitness landscape, where the initial transition step $010 \rightarrow 011$ has a path flexibility of 2 , since the global optimum 101 can be reached either via path $011 \rightarrow 001 \rightarrow 101$ or via path $011 \rightarrow 111 \rightarrow 101$. Path flexibility can also be expressed in graph-theoretical terms. The path flexibility of a node regarding a specific local optimum is the number of paths of directed links between this node and the local optimum. The Appendix provides the formal expression.

The third flexibility measure is not based on uncertainty due to changing evidence but on uncertainty caused by changing preferences. In our discussion so far, we reasoned from a single fitness function, for example, a 'social welfare function'. Such reasoning presupposes that consensus exists in a society on how alternative technologies should be assessed. However, more often than not, different social groups have different valuations of a technology (Pinch and Bijker 1984). For example, some actors prefer a future car system that is less expensive than the current one. Other actors may emphasize that a future car system should be less polluting, even if the costs of such a system would be higher than the costs of the current system. We take multiple preference sets into account by viewing technological change as taking place in a single design space with multiple fitness functions attached to it. 
Fig. 2 An alternative fitness function $g$

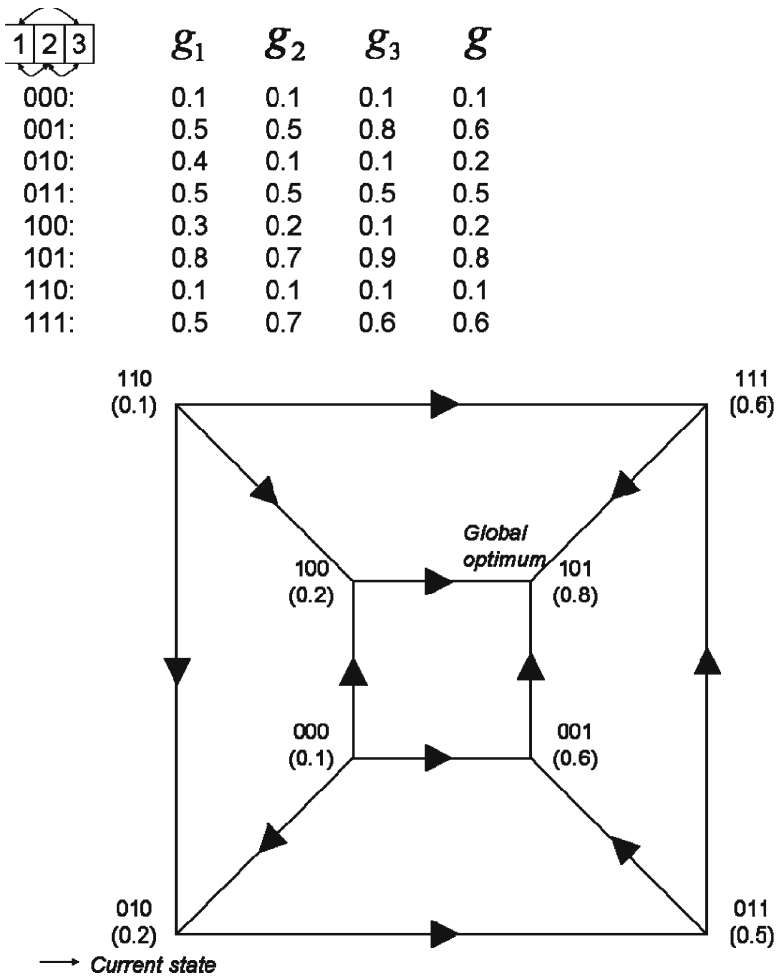

In Fig. 2, an alternative set of fitness values is presented for the same design space as in Fig. 1 reflecting the valuation of a second social group. We can now define a flexible initial transition step as a step that meets both sets of preferences at the same time, i.e., a transition step that increases both fitness $f$ and fitness $g$. Thus, if the initial transition step is described as $x \rightarrow y$, a flexible strategy is one which holds that both $f(x) \geq f(y)$ and $g(x) \geq g(y)$. In the examples of Figs. 1 and 2, such a strategy would lead to the path: $010 \rightarrow 011$. One could call this strategy a win-win strategy, also known as a Pareto improvement. Such a strategy is flexible in the sense that, if the power balance between different groups having different preferences shifts over time (e.g., a change in government), it will not lead the decision-maker to undo the initial transition step and to reverse the transition process. We call this type of flexibility of the initial transition step preference flexibility.

\section{The transition towards a sustainable car system}

In the remainder, we will apply the three flexibility measures to data on fitness values of alternative future car systems. A transition path from the current 
oil-based individual transportation system to a more sustainable system meets the assumptions underlying the framework just described:

- Complexity: the system is complex in that it consists of subsystems, which function interdependently but can be changed independently

- Uncertainty: the fitness of alternative designs can be assessed in principle ex ante, but is highly uncertain

- Myopia: any technological transition will most likely occur in a series of myopic rather than coordinated transition steps in subsystems, because any change in a sub-system is a very costly and lengthy process

- Multiple preferences: different social groups apply different evaluation criteria to assess the desirability of alternative options

In order to apply our flexibility measures to assess alternative transition steps towards a future sustainable car system, we proceed as follows. First, we construct the design space of alternative future car systems. We then assign to each alternative design one fitness value reflecting the energy efficiency as a proxy of its economic performance and one fitness value reflecting the reduction in greenhouse gas (GHG) emissions as a proxy for climate performance. We assume there are two dominant social groups with different preferences, with one group aiming to reduce the energy requirements of cars (economic) and another group aiming to reduce GHG emissions (climate). For both fitness criteria, we derive the global and local optima. Then, we compute the three flexibility measures for each up-hill initial transition step. From the results, we draw conclusions regarding the desirability of alternative transition steps.

\subsection{Design space}

Technology assessment studies regarding future car systems generally use a well-to-wheel (WTW) perspective to describe an alternative technological system in terms of its subsystems. We use data from previous WTW studies to build the design space. To classify each of the subsystems, a high level of aggregation is chosen, thus ignoring sub-sub-systems within each subsystem. Based on previous studies, we distinguish five major subsystems following the WTW logic: the energy source (seven options); the application of carbon capture and sequestration (CCS; two options); process scale, process location and distribution to filling station (seven options); car fuel (nine options); and vehicle type (three options). The WTW system including all options per subsystem is shown in Fig. 3.

All possible combinations between alternative subsystem designs form the WTW design space representing possible future car systems. Even for this high level of aggregation with only five subsystems, there already exist $7 \cdot 2 \cdot 7 \cdot 9 \cdot 3=$ 2,646 theoretical combinations of energy sources, CCS, distribution systems, fuels and vehicles. In this design space, the current dominant design is classified as crude oil, no CCS, large, centralized truck distribution, gasoline fuel and internal combustion engine. 

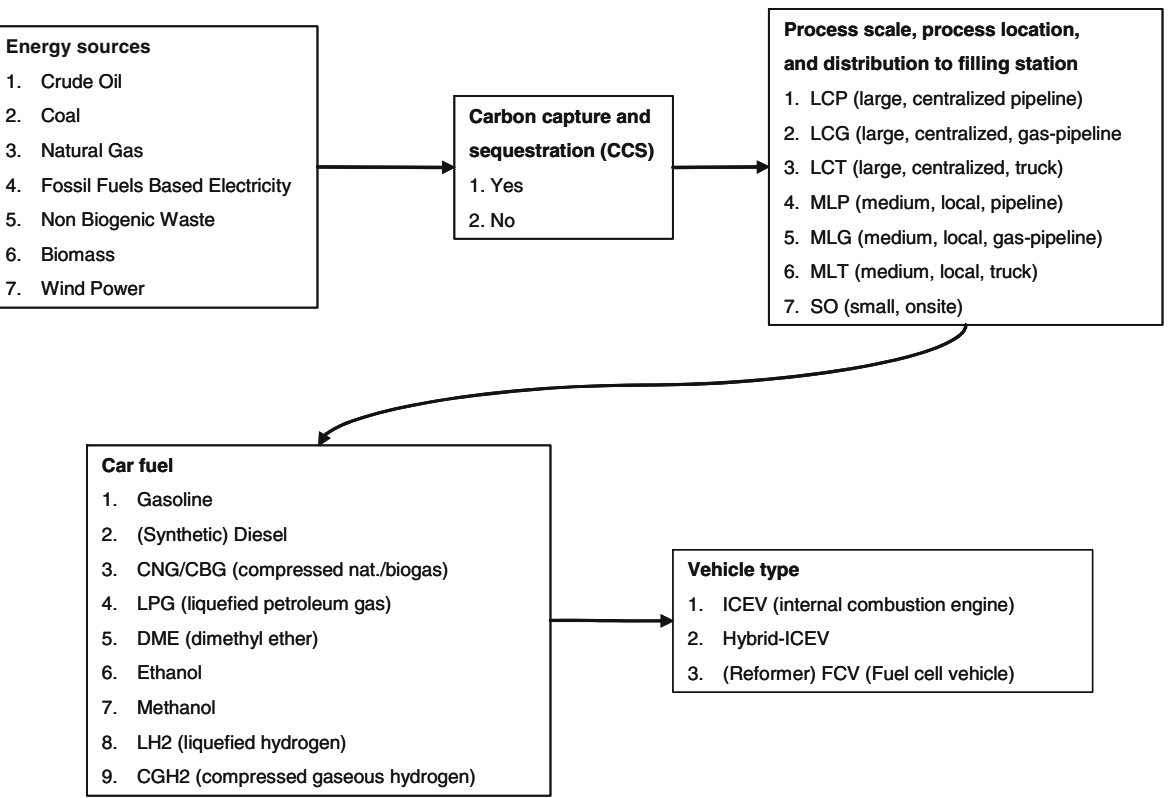

Fig. 3 Subsystems of the WTW system

\subsection{Fitness landscapes}

We assess the fitness (or performance) of each WTW system in two dimensions reflecting two preferences: WTW energy requirements per kilometer driven and WTW GHG emissions per kilometer driven. The first we call efficiency fitness and the second climate fitness. Efficiency fitness is important given concerns about the availability and increasing prices of (fossil) fuel resources, whereas climate fitness is important given concerns about the negative climate impact of our current fossil-fuel based car system. The two fitness values of each possible technological system are taken from Schwoon (2006), which in turn is based on the WTW analyses available (GM et al. 2002; Ahlvik and Brandberg 2001; EC-JRC 2006).

Only a minority of 987 out of the 2,646 designs have a fitness value equal to or higher than the fitness value of the current dominant design in terms of one or both fitness criteria. In order to find the local optima, we check which designs could not be improved by a change in a single subsystem regarding efficiency fitness or climate fitness. As the fitness values are rounded, neighboring designs with identical performance occur. ${ }^{5}$ Thus, optima can consist of more than one design, which are neighboring in the sense that they are no more than

\footnotetext{
${ }^{5}$ Schwoon (2006) choose to round fitness values to account for some of the uncertainty in the technical data on the expected performance of each technology. Note that rounding means that agents engage in what is called "noisy search" (Levinthal 1997).
} 
Table 1 Optima of WTW systems

\begin{tabular}{|c|c|c|c|c|c|}
\hline & $\begin{array}{l}\text { Current } \\
\text { system }\end{array}$ & $\begin{array}{l}\text { Global optimum } \\
\text { with regard to } \\
\text { efficiency }\end{array}$ & $\begin{array}{l}\text { Local optimum } \\
A \text { with regard } \\
\text { to efficiency }\end{array}$ & $\begin{array}{l}\text { Local optimum } \\
B \text { with regard } \\
\text { to efficiency }\end{array}$ & $\begin{array}{l}\text { Global optimum } \\
\text { with regard to } \\
\text { environment }\end{array}$ \\
\hline Energy source & Crude Oil & Crude Oil & Wind power & NG & Biomass \\
\hline $\mathrm{CCS}$ & No & No & No & No & Yes \\
\hline Distribution & LCT & LCG or LCT & MLT & $\begin{array}{l}\text { LCG } \text { or LCT or } \\
\text { MLG or MLT } \\
\text { or SO }\end{array}$ & LCT \\
\hline Fuel & gasoline & $\mathrm{CGH} 2$ & LH2 & $\mathrm{CNG}$ & LH2 \\
\hline Vehicle & ICEV & FCV & FCV & Hybrid-ICEV & $\begin{array}{l}\text { ICEV or } \\
\text { Hybrid-ICEV } \\
\text { or FCV }\end{array}$ \\
\hline
\end{tabular}

one transition step away from each other. Table 1 contains a list of local optima in the WTW design space.

What is clear from Table 1 is that the three optima with regard to energy efficiency are quite different and thus represent truly different futures. All three local optima differ in at least three dimensions from one another. Strikingly, only one optimum was found when assessing alternative designs with regard to climate performance. This design also differs from the three optima regarding energy efficiency in at least three dimensions. We can thus conclude that the two performance measures are conflicting targets. A technological transition driven by economic performance would therefore look very different from a transition driven by climate performance.

\subsection{Flexibility results}

The three flexibility measures we distinguished in the previous section refer to the flexibility of an initial transition step. The possible initial transition steps are derived by checking which transition steps would not decrease either efficiency fitness or climate fitness starting from the current system being crude oil, no CCS, large, centralized truck distribution (LCT), gasoline fuel and internal combustion engine. In the economic fitness landscape, there is a choice between four initial transition steps:

- changing the distribution system into a large, centralized pipeline system (LCP)

- changing the dominant fuel into diesel

- changing the vehicle technology into a hybrid vehicle (hybrid-ICEV)

- changing the vehicle technology into a fuel cell vehicle (FCV)

In the climate fitness landscape, there is a choice between seven initial steps:

- introducing carbon capture and sequestration (CCS)

- changing the distribution system into a large, centralized pipeline system (LCP)

- changing the dominant fuel into diesel

- changing the dominant fuel into liquefied petroleum gas $\left(\mathrm{LH}_{2}\right)$ 
Table 2 Design flexibility of optima

\begin{tabular}{|l|l|l|l|l|}
\hline & \multicolumn{4}{|c|}{ Design flexibility } \\
\hline First transition step & $\begin{array}{l}\text { Global } \\
\text { optimum } \\
\text { efficiency }\end{array}$ & $\begin{array}{l}\text { Local optimum } \\
\text { A } \\
\text { efficiency }\end{array}$ & $\begin{array}{l}\text { Local optimum } \\
\text { efficiency }\end{array}$ & $\begin{array}{l}\text { Global } \\
\text { optimum } \\
\text { environment }\end{array}$ \\
\hline Transition to CCS & & & & \\
\hline Transition to LCP & & & out of reach $^{a}$ & \\
\hline Transition to Diesel & & & & \\
\hline Transition to $\mathrm{LH}_{2}$ & & & & \\
\hline Transition to $\mathrm{CGH}$ & & & & \\
\hline Transition to $\mathrm{Hybrid-ICEV}$ & & & & \\
\hline Transition to FCV & & & & \\
\hline
\end{tabular}

${ }^{\text {a }}$ This optimum can be reached, but not within five steps as required

- changing the dominant fuel into compressed gaseous hydrogen $\left(\mathrm{CGH}_{2}\right)$

- changing the vehicle technology into a hybrid vehicle (hybrid-ICEV)

- changing the vehicle technology into a fuel cell vehicle (FCV)

Note that the four initial transition steps that are acceptable in the economic fitness landscape are also acceptable in the climate fitness landscape. We thus have seven possible initial transition steps.

We compute the flexibility measures regarding each of the seven possible initial transition steps. In the computations, we take into account only those transition paths towards optima that are not greater than five steps, including the initial step. We thus assume that transition paths involving more than five transition steps are beyond the relevant time horizon of society.

The first type of flexibility distinguished above, called design flexibility, we define as the number of different optima that can be reached after the initial transition step has taken place. With regard to the climate fitness landscape, this form of flexibility is irrelevant because only one optimum is present in the climate fitness landscape. With regard to the economic fitness landscape, Table 2 shows that all four possible initial transition steps remain flexible with regard to all three optima. However, a transition step to LCP excludes the possibility to reach local optimum $B$ because such a path requires more than the maximum five transition steps we have imposed.

The second flexibility measure called path flexibility counts the number of different paths that lead to an optimum given the initial transition step and given the restriction of a maximum path length of five transition steps. These results are given in Table 3 . First, we observe that the global optima can be reached via many more paths than the local optima. This can be interpreted as an indication that chances of a lock-in in a sub-optimal system due to current decisions are rather low. ${ }^{6}$ Second, we observe striking differences for path

\footnotetext{
${ }^{6}$ This outcome is not surprising because it is known from simulations of the NK-model that the size of the 'basin of attraction' of an optimum is positively related to the fitness of that optimum (Kauffman 1993).
} 
Table 3 Path flexibility of optima

\begin{tabular}{|c|c|c|c|c|}
\hline \multirow{2}{*}{$\begin{array}{l}\text { First transition } \\
\text { step }\end{array}$} & \multicolumn{4}{|l|}{ Path flexibility } \\
\hline & $\begin{array}{l}\text { Global optimum } \\
\text { efficiency }\end{array}$ & $\begin{array}{l}\text { Local optimum } \\
A \text { efficiency }\end{array}$ & $\begin{array}{l}\text { Local optimum } \\
B \text { efficiency }\end{array}$ & $\begin{array}{l}\text { Global optimum } \\
\text { environment }\end{array}$ \\
\hline Transition to CCS & & & & 50 \\
\hline Transition to LCP & 11 & 1 & Out of reach & 1 \\
\hline Transition to Diesel & 14 & 1 & 2 & 59 \\
\hline Transition to $\mathrm{LH}_{2}$ & & & & 47 \\
\hline Transition to $\mathrm{CGH}_{2}$ & & & & 11 \\
\hline $\begin{array}{l}\text { Transition to } \\
\text { Hybrid-ICEV }\end{array}$ & 15 & 2 & 7 & 32 \\
\hline Transition to FCV & 27 & 5 & 4 & 22 \\
\hline
\end{tabular}

flexibility regarding different optima and regarding different initial transition steps. For an initial step to diesel as the dominant fuel, we even counted as many as 59 possible paths leading to the global optimum in the climate fitness landscape. Such high numbers are related to the rounding of fitness values as mentioned above. Rounding creates fitness plateaus which can be traversed via many different routes.

In order to get more insight in the relevant path flexibility of each initial transition step, we repeated the exercise on path flexibility, taking into account only the shortest path. Table 4 shows that the path flexibility drastically decreases if only shortest paths are counted. Both for global and local optima, it holds that often only one possible path exists to reach the particular design. One can also observe that the shortest paths to the global optima are generally shorter than the shortest paths to local optima.

Finally, we assess each transition step on the basis of preference flexibility. All initial transition steps towards an optimum in the economic fitness landscape are also transition steps towards the global optimum in the climate fitness landscape. However, the reverse is not the case, as three out of seven initial transition steps towards the climate optimum do not lie on a path towards an energy efficiency optimum. If a decision-maker would only prefer emission reductions, a switch to CCS or a switch to $\mathrm{CGH}_{2}$ or a switch to $\mathrm{LH}_{2}$ would directly imply a significant decrease in energy efficiency. In that case,

Table 4 Path flexibility of optima for shortest paths only

\begin{tabular}{|c|c|c|c|c|}
\hline \multirow{2}{*}{$\begin{array}{l}\text { First transition } \\
\text { step }\end{array}$} & \multicolumn{4}{|c|}{ Path flexibility (shortest paths only) } \\
\hline & $\begin{array}{l}\text { Global optimum } \\
\text { efficiency }\end{array}$ & $\begin{array}{l}\text { Local optimum } \\
A \text { efficiency }\end{array}$ & $\begin{array}{l}\text { Local optimum } \\
B \text { efficiency }\end{array}$ & $\begin{array}{l}\text { Global optimum } \\
\text { environment }\end{array}$ \\
\hline Transition to CCS & & & & 1 (3 steps) \\
\hline Transition to LCP & 1 (4 steps) & 1 (5 steps) & out of reach & 1 (5 steps) \\
\hline Transition to Diesel & 1 (3 steps) & 1 (5 steps) & $2(5$ steps $)$ & $6(4$ steps $)$ \\
\hline Transition to $\mathrm{LH}_{2}$ & & & & 2 (4 steps) \\
\hline Transition to $\mathrm{CGH}_{2}$ & & & & 1 (3 steps) \\
\hline Transition to Hybrid-ICEV & 3 (3 steps) & 2 (5 steps) & 1 (4 steps) & 3 (4 steps) \\
\hline Transition to FCV & 1 (2 steps) & 1 (4 steps) & 4 (5 steps) & 3 (4 steps) \\
\hline
\end{tabular}

Length of shortest path between brackets 
these choices have a regret potential. A future change in preference towards reducing energy requirements may well undo these steps, thus wasting time and resources. The remaining four initial transition steps are flexible with regard to a change in preferences. From Table 4, it can further be observed that the shortest paths to the global optimum regarding climate performance (taking an initial step to either $\mathrm{CCS}$ or $\mathrm{CGH}_{2}$ ) would come at the cost of lowering energy efficiency. Thus, the fastest route to the climate optimum is incompatible with energy efficiency improvement.

If one considers the results on the three flexibility measures jointly, some initial transition steps seem more desirable than others. The three initial steps that do not lie on a path towards an energy efficiency optimum (initial steps to $\mathrm{CCS}, \mathrm{LH}_{2}$ or $\mathrm{CGH}_{2}$ ) are undesirable, as energy efficiency would decrease. Out of the four remaining options, changes in the vehicle technology towards either a hybrid car or a fuel cell car seem most desirable because of their combined scores on design flexibility and path flexibility.

\section{Summary and conclusions}

We presented a methodology to transition management that focuses on the transition path instead of the end state of a transition. More specifically, we used a complex systems approach to analyze the desirability of potential initial transition steps, given that technical evidence and social preferences may change in the future. The approach we proposed is a general approach that can be applied to any complex technology for which data on fitness values is available.

Applying the framework to data on economic and climate performance of alternative car systems, we found that design flexibility is high for all initial transition steps in that optima can still be reached after the initial step. Regarding path flexibility, defined as the number of different paths leading to an optimum, we found considerable differences across initial transition steps. Another finding was that global optima can be reached via many more paths than local optima, which reduces the chances of a lock-in into a system that is considered sub-optimal at present. Finally, we found that all initial steps acceptable in the energy efficiency landscape are also acceptable in the climate fitness landscape without the reverse being true. This renders initial transition steps robust for a change in preference from energy efficiency to environment. However, vice versa, not all initial transition steps were found to be robust for a change in preference from climate to energy efficiency. In addition to efficiency and climate fitness, the proposed methodology makes it possible to assess possible transition steps with respect to other possible fitness measures as well given the availability of sufficiently detailed performance data.

Overall, we conclude that changes in the vehicle technology towards either a hybrid car or a fuel cell car are most desirable because these are flexible in all respects. However, it should be borne in mind that the empirical application in first instance served an illustrative purpose. The conclusions that can be 
derived from the exercise should be treated cautiously, because of the lack of precise technical data for this particular case (Schwoon 2006).

The proposed methodology can be extended to contexts where alternative search strategies apply. Instead of myopic search involving changes in a single subsystem only, larger search distances-involving changes in multiple subsystems simultaneously-apply in many technology contexts (Auerswald et al. 2000; Kauffman et al. 2000). Such an extension may well yield different results for our flexibility measures, yet without changing their meaning in any fundamental sense.

The case study illustrated that the methodology developed in this paper can lead to useful insights regarding optimal transition strategies. Taking into account future uncertainty and the flexibility of transition steps leads to more robust transition strategies, improving the chances of completing a desired transition. The methodology we proposed is complementary to alternative decision-theoretic approaches, such as real option theory, which are based on more stringent assumptions about agents' computational power and the information available to them (Trigeorgis 1996). Furthermore, our prospective approach can supplement other empirical methodologies in the field of transition management (Kemp 1994; Geels 2002; Hekkert et al. 2007; Markard and Truffer 2008) as well as simulation models on technological transitions (Windrum and Birchenhall 2005; Van den Bergh 2007; Faber and Frenken 2009; Struben and Sterman 2008).

Acknowledgements We would like to thank two reviewers and the participants of the DRUID Summer conference (Copenhagen, 2007), the workshop on 'Computational and Mathematical Approaches to Societal Transitions' (Leiden, 2007), the workshop on 'Demand, Product Characteristics and Innovation' (Jena, 2007) and the workshop on 'Empirical Analyses of Environmental Innovations' (Karlsruhe, 2008) for useful comments. The paper does not represent the view of Statkraft in any way. The usual disclaimer applies.

\section{Appendix}

A fitness landscape can be represented by a graph $D=(V, A)$ that consists of a set of nodes $V$ (the design space) and a set of directed arcs $A$ between neighboring states $x$ and $y$ (the transition steps). For each pair of neighboring states, we have a directed arc $a(x, y) \in A$, where $a(x, y)=x \rightarrow y$ if $f(y) \geq$ $f(x)$. The set of arcs is given by:

$$
A=\{(x \rightarrow y)\}:(f(y) \geq f(x)) \wedge(x, y \in V)
$$

where $f$ represents the fitness function. 
The optima in the fitness landscape can be described as follows:

Global optimum: $\quad x \in V \quad$ s.t. $\neg \exists y \in V: f(y)>f(x)$ Local optimum: $\quad x \in V$ s.t. $\neg \exists y \in V:(f(y)>f(x)) \wedge(a(x, y) \in A)$

Note that each global optimum is thus also a local optimum. The design flexibility of an initial transition step is then defined by the number of different local optima in $O \subset V$ that can be reached within a certain number of later transition steps. This design flexibility $F^{d}$ given an initial transition step $a\left(x_{0}, x_{1}\right)$ is then given by:

$$
\begin{aligned}
F^{\mathrm{d}}= & |R \subset O|:\left\{\left(x_{n} \in O\right) \wedge\left(\left\{x_{0}, x_{1}, \ldots, x_{n}\right\} \subset O\right)\right. \\
& \left.\wedge\left(\left\{a\left(x_{o}, x_{1}\right), a\left(x_{1}, x_{2}\right), \ldots, a\left(x_{n-1}, x_{n}\right)\right\} \subset A\right)\right\}
\end{aligned}
$$

Where $n$ is total number of steps in a transition path. In our computations, we used a maximum allowed path length of $n=5$.

The second measure of flexibility describes the number of different paths that lead to a local optimum $x_{n}$. This flexibility with regard to the number of paths $F^{p}$ given an initial step $a\left(x_{0}, x_{1}\right)$ and leading to local optimum $x_{n}$ is then defined by:

$$
\begin{aligned}
F^{\mathrm{p}}= & |P \subset A|:\left\{\left(\left\{x_{0}, x_{1}, \ldots x_{n}\right\} \subset V\right)\right. \\
& \left.\wedge\left(\left\{a\left(x_{0}, x_{1}\right), a\left(x_{1}, x_{2}\right), \ldots, a\left(x_{n-1}, x_{n}\right)\right\} \subset A\right)\right\}
\end{aligned}
$$

\section{References}

Ahlvik P, Brandberg A (2001) Well-to-wheel efficiency for alternative fuels from natural gas or biomass. Swedish National Road Administration. www.vv.se/publ_blank/bokhylla/ miljo/lista.htm

Altenberg L (1994) Evolving better representations through selective genome growth. In: Proceedings of the IEEE world congress on computational intelligence, pp 182-187

Arthur WB (1989) Competing technologies, increasing returns, and lock-in by historical events. Econ J 99:116-131

Auerswald P, Kauffman SA, Lobo J, Shell K (2000) The production recipes approach to modeling technological innovation: an application to learning by doing. J Econ Dyn Control 24:389-450

Cowan R (1990) Nuclear power reactors: a study in technological lock-in. J Econ Hist 50:541-567

David PA (1985) The economics of QWERTY. Am Econ Rev (Papers and Proceedings) 75: $332-337$

EC-JRC (European Commission - Joint Research Center) (2006) Well-to-wheels analysis of future automotive fuels and powertrains in the European context, Well-to-Wheels Report, Version 2b, May 2006. http://ies.jrc.ec.europa.eu/WTW

Faber A, Frenken K (2009) Models in evolutionary economics and environmental policy. Towards an evolutionary environmental economics. Technol Forecast Soc Change 76(4):462-470. doi:10.1016/j.techfore.2008.04.009

Frenken K (2006) Innovation, evolution and complexity theory. Edward Elgar, Cheltenham

Geels FW (2002) Technological transitions as evolutionary reconfiguration processes: a multi-level perspective and a case-study. Res Policy 31:1257-1274

GM, LBST, BP, ExxonMobil, Shell, TotaFinaElf (2002) Well-to-wheel analysis of energy use and greenhouse gas emissions of advanced fuel/vehicle systems - a European study. http://www.lbst.de/ 
Hekkert MP, Suurs R, Negro SO, Smits REHM, Kuhlmann S (2007) Functions of innovation systems: a new approach for analyzing technological change. Technol Forecast Soc Change 74:413-432

Hughes TP (1983) Networks of power: electrification in western society, 1880-1930. Johns Hopkins University Press, Baltimore

Kauffman SA (1993) The origins of order. Self-organization and selection in evolution. Oxford University Press, New York

Kauffman SA, Lobo J, Macready WG (2000) Optimal search on a technology landscape. J Econ Behav Organ 43:141-166

Kemp R (1994) Technology and the transition to environmental sustainability. Futures 26: 1023-1046

Levinthal DA (1997) Adaptation on rugged landscapes. Manag Sci 43:934-950

Markard J, Truffer B (2008) Technological innovation systems and the multi-level perspective: towards an integrated framework. Res Policy 37:596-615

Pinch T, Bijker WE (1984) The social construction of facts and artifacts: or how the sociology of science and the sociology of technology might benefit each other. Soc Stud Sci 14:399-441

Rosenberg N (1969) The direction of technical change: inducement mechanisms and focussing devices. Econ Dev Cult Change 18:1-24

Saviotti PP, Metcalfe JS (1984) A theoretical approach to the construction of technological output indicators. Res Policy 13:141-151

Schwoon M (2006) Managing the transition to hydrogen and fuel cell vehicles - insights from agent-based and evolutionary models. Reports on earth system science, vol 32. Max Planck Institute for Meteorology, Hamburg

Silverberg G, Verspagen B (2005) A percolation model of innovation in complex technology spaces. J Econ Dyn Control 29:225-244

Simon HA (1969) The sciences of the artificial. MIT, Cambridge (3rd edn, 1996)

Simon HA (2002) Near decomposability and the speed of evolution. Ind Corp Change 11:587-599

Struben J, Sterman JD (2008) Transition challenges for alternative fuel vehicle and transportation systems. Environ Plann B Plann Des 35(6):1070-1097. doi:10.1068/b33022t

Taylor JWR (1995) Constraints impeding the commercial use of helicopters. In: Leary WM (ed) From airships to airbus. The history of civil and commercial aviation. Volume 1: Infrastructure and environment. Smithsonian Institution Press, Washington \& London, pp 155-167

Trigeorgis L (1996) Real options: managerial flexibility and strategy in resource allocation. The MIT Press, Cambridge

Van den Bergh J (2007) Evolutionary thinking in environmental economics. J Evol Econ 17: $521-549$

Vincenti W (1990) What engineers know and how they know it. Johns Hopkins University Press, Baltimore

Windrum P, Birchenhall C (2005) Structural change in the presence of network externalities: a co-evolutionary model of technological successions. J Evol Econ 15:123-148 